\title{
Observation of a Chirality-Induced Exchange-Bias Effect
}

\author{
K. Chen $\odot,{ }^{1,{ }^{*}}$ A. Philippi-Kobs, ${ }^{2}$ V. Lauter, ${ }^{3}$ A. Vorobiev, ${ }^{4}$ E. Dyadkina,${ }^{5}$ V.Yu. Yakovchuk, ${ }^{6}$ \\ S. Stolyar, ${ }^{6,7}$ and D. Lott $^{8}$ \\ ${ }^{1}$ Helmholtz-Zentrum Berlin für Materialien und Energie, Albert-Einstein-Straße 15, 12489 Berlin, Germany \\ ${ }^{2}$ Deutsches Elektronen-Synchrotron DESY, Notkestraße 85, 22607 Hamburg, Germany \\ ${ }^{3}$ Neutron Scattering Division, Neutron Sciences Directorate, Oak Ridge National Laboratory, Oak Ridge, \\ Tennessee 37831, USA \\ ${ }^{4}$ Department of Physics and Astronomy, Uppsala University, Box 516, SE-75120 Uppsala, Sweden \\ ${ }^{5}$ Swiss-Norwegian Beamlines, European Synchrotron Radiation Facility, 38043 Grenoble, France \\ ${ }^{6}$ Siberian Federal University, Krasnoyarsk, 660041, Russia \\ ${ }^{7}$ Kirensky Institute of Physics SB RAS, Krasnoyarsk, 660036, Russia \\ ${ }^{8}$ Institute for Materials Research, Helmholtz-Zentrum Geesthacht, Max-Planck Straße 1, 21502 Geesthacht, \\ Germany
}

(Received 1 February 2019; revised manuscript received 24 May 2019; published 22 August 2019)

\begin{abstract}
Chiral magnetism that manifests in the existence of skyrmions or chiral domain walls offers an alternative way for creating anisotropies in magnetic materials that might have large potential for application in future spintronic devices. Here we show experimental evidence for an alternative type of in-plane exchange-bias effect present at room temperature that is created from a chiral $90^{\circ}$ domain wall at the interface of a ferrimagnetic-ferromagnetic Dy-Co/Ni-Fe bilayer system. The chiral interfacial domain wall forms due to the exchange coupling of $\mathrm{Ni}-\mathrm{Fe}$ and Dy-Co at the interface and the presence of Dzyaloshinskii-Moriya interaction in the Dy-Co layer. As a consequence of the preferred chirality of the interfacial domain wall, the sign of the exchange-bias effect can be reversed by changing the perpendicular orientation of the Dy-Co magnetization. The chirality-created tunable exchange bias in Dy-Co/ $\mathrm{Ni}-\mathrm{Fe}$ is very robust against high in-plane magnetic fields $\left(\mu_{0} H \leq 6 \mathrm{~T}\right)$ and does not show any aging effects. Therefore, it overcomes the limitations of conventional exchange-bias systems.
\end{abstract}

DOI: 10.1103/PhysRevApplied.12.024047

\section{INTRODUCTION}

Since the discovery of the exchange-bias (EB) effect in the 1950s by Meiklejohn and Bean when they were studying Co particles embedded in their native antiferromagnetic oxide [1], it has been intensively investigated due to its importance in magnetic spin valves and their applications in data-storage technology [2,3]. Despite its wide application, the EB effect is still not completely understood due to the technological limitations involved in directly observing and manipulating spin structures at an atomically thin magnetic interface [4,5]. It is generally considered to form from an uncompensated spin configuration at the ferromagnetic-antiferromagnetic interface with both frozen and rotatable spins [6-10]. Besides the classic system, EB-related effects have also been observed in ferrimagnetic-based materials with compensated interfaces [11-17]. Moreover, an atomic EB has been reported in a single ferrimagnetic $\mathrm{DyCo}_{4}$ thin film where the magnetic surface region pins the magnetization of the bulk [18].

\footnotetext{
*kaichen.hzg@gmail.com
}

Recently, chiral ferrimagnetism was observed in amorphous Gd-Co films [19] due to the interfacial Dzyaloshinskii-Moriya-interaction (DMI) [20,21]. A new class of EB effects based on orthogonally-exchangecoupled systems has been proposed and observed [2226]. There, the DMI is suggested as a mechanism to drive the EB in G-type antiferromagnetic perovskite interfaces [22] as well as in an antiferromagnetic-ferromagnetic $\mathrm{IrMn}_{3} / \mathrm{Co}(111)$ bilayer [23] or is considered to be responsible for forming a tunable EB effect in dilute magnetic alloys [24]. A new form of magnetic anisotropy, originating from the tilted magnetization at the edges of magnetic structures due to the DMI, has been investigated in $\mathrm{MgO} /(\mathrm{Co}, \mathrm{Fe}) \mathrm{B} / \mathrm{Pt}$ [25] and $\mathrm{Pt} / \mathrm{Co} / \mathrm{Ir}$ [26]. In the latter case, an out-of-plane EB-like effect was found when an additional in-plane magnetic field was used. In a similar way, a chirality-induced asymmetric domain nucleation in $\mathrm{Pt} / \mathrm{Co} / \mathrm{AlO}_{x}$ with perpendicular magnetic anisotropy was observed when in-plane magnetic fields were used [27]. The latter studies demonstrate effective paths for using DMI and DMI-like effects for creating and manipulating EB effects with high potential for future applications. 
Moreover, the presence of DMI in magnetic thin film systems can lead to the creation of chiral domain walls (DWs), which enable a surprisingly fast current-driven DW motion [28-35], while the velocity and the direction can be controlled by interfacial engineering [35-37].

Here we report an approach to create a robust and tunable EB effect at room temperature that is based on a chiral interfacial domain wall (approximately $90^{\circ}$ ) present at the interface of orthogonally coupled layers of ferrimagnetic Dy-Co and ferromagnetic Ni-Fe. Superconducting-quantum-interference-device (SQUID), magneto-optical-Kerr-effect (MOKE), and polarizedneutron-reflectivity (PNR) measurements confirm the presence of an interfacial domain wall with a preferred chirality whose origin can be explained by the presence of the DMI effect in the Dy-Co layer.

\section{CONCEPT OF CHIRALITY-CREATED EXCHANGE BIAS}

The magnetization profile in a bilayer system strongly depends on the magnetic anisotropies present in the layers and the nature of the magnetic interactions occurring at the interface. In Fig. 1(a), a bilayer system consisting of two planar films with uniaxial magnetic anisotropies is considered whose easy axes (as marked by $K_{u x}$ and $K_{u z}$ ) are oriented perpendicular to each other. The exchange coupling at the interface results in a slightly tilted effective easy axis as illustrated for the top layer in Fig. 1(a). Such tilted magnetic configurations were experimentally observed, for example, in orthogonally-exchange-coupled $[\mathrm{Co} / \mathrm{Pd}] / \mathrm{Ni}-\mathrm{Fe}$ and $\mathrm{Co}-\mathrm{Pt} / \mathrm{Ni}-\mathrm{Fe}$ exchange spring systems $[38,39]$, or were obtained by resputtering processes [40] or by using curved surfaces as substrates $[41,42]$. The coupling between both layers will lead to four energetically equivalent interfacial spin textures, marked as 1,2,3, and 4 , with their spins from top to bottom aligned as up right, down left, down right, and up left. In the case of a pinning of the magnetization component $M_{\mathrm{top}, z}$ of the top layer along one of the out-of-plane directions, a two-step hysteresis loop will be observed when a magnetic field is applied along the $x$ axis [Fig. 1(d)]. Such a two-step hysteresis loop as well as a training effect of the exchange bias has been observed in coupled ferromagnetic bilayer structures of $\mathrm{Co}-\mathrm{Cr} / \mathrm{Ru} / \mathrm{Co}-\mathrm{Pt}-\mathrm{Cr}-\mathrm{B}$. [43].

We propose a way to form a tunable exchange-bias effect by lifting the degeneracy between the states with different chirality of the interfacial domain wall. The chirality refers here to the clockwise or counterclockwise spin rotation from the top layer to the bottom layer [clockwise for states 1 and 2, Fig. 1(b); counterclockwise for states 3 and 4, Fig. 1(c)]. Such chiral domain walls can originate from
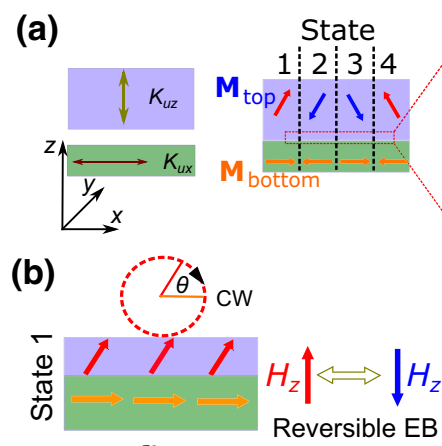

(c)

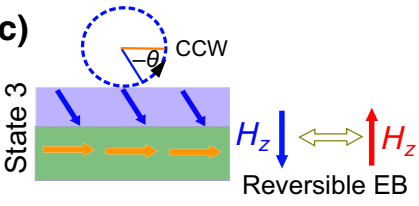

$\begin{array}{llll}1 & 2 & 3 & 4\end{array}$
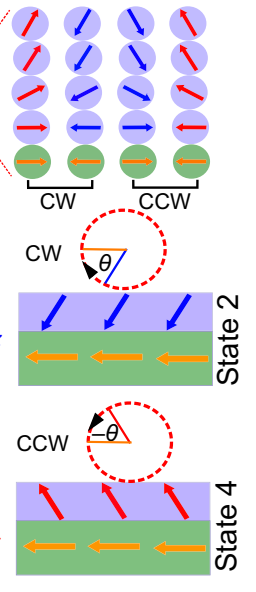

(d)
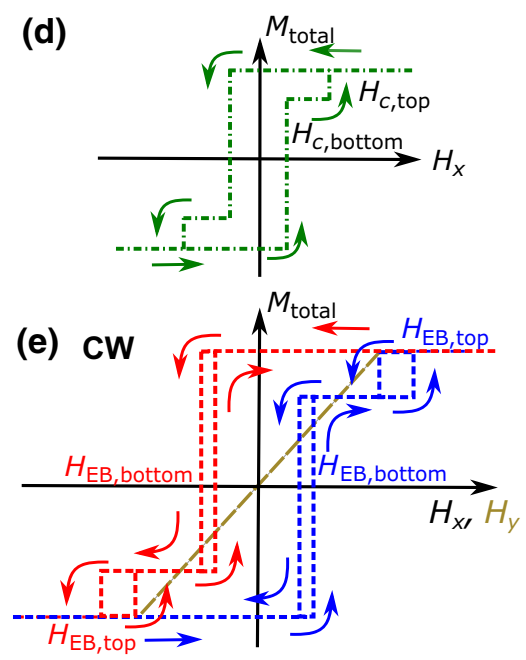

FIG. 1. Exchange-bias effect created from a chiral interfacial domain wall. The figure illustrates the magnetization profile in remanence and magnetization reversal along the $x$ axis for an exchange coupled bilayer system whose magnetic anisotropies are perpendicular to each other:(a) The uniaxial magnetic anisotropies (represented by the anisotropy constants $K_{u x}$ and $K_{u z}$ ) and ferromagnetic exchange coupling at the interface result in a two step hysteresis when the magnetic field is applied along the $x$ axis (d). In the presence of DMI at the interface, the four interfacial spin textures (marked as 1,2,3, and 4) become energetically unequal and a preferred (b) clockwise (CW) or (c) counterclockwise (CCW) spin rotation from the top layer to the bottom layer exists (represented by a positve or negative angle $\theta$ ). (e) As shown for the CW scenario, the DMI effect induces a dual EB effect for the switching of

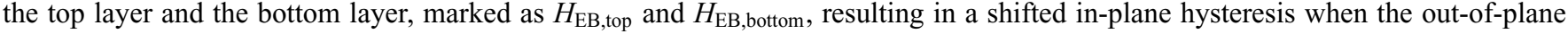
magnetization of the top layer is fixed. The sign of the in-plane EB effects can be switched by an out-of plane magnetic field $H_{z}$ that changes the orientation of $M_{z}$ of the top layer. In the case of a favorable CW spin rotation, a negative (red) or positive (blue) shift of the $M_{x}\left(H_{x}\right)$ hysteresis exists for a downward or upward magnetization $M_{z}$ of the top layer, while no shift (brown) is expected for the $M_{y}\left(H_{y}\right)$ hysteresis. 
the bulklike DMI [20,21] or the DMI due to the breaking of the inversion symmetry at the interface. The DMI favors spins on neighboring sites to be aligned orthogonally, and thus the interplay between DMI and exchange coupling between both layers at the interface will lead to noncollinear spin textures with a preferred chirality. Depending on the orientation of the perpendicular component of magnetization in the top layer, $M_{\mathrm{top}, z}$, a unidirectional shift of the hysteresis loop occurs [Fig. 1(e)]. Hence, the direction of the EB shift can be controlled by setting $M_{\mathrm{top}, z}$ with a sufficiently strong out-of-plane magnetic field.

\section{CHIRALITY-CREATED EXCHANGE-BIAS EFFECT IN Dy-Co/Ni-Fe}

To realize a chiral interfacial domain wall in an orthogonally coupled system and to create a tunable EB as shown in Fig. 1(e), a bilayer consisting of a ferromagnetic $\mathrm{Ni}_{80} \mathrm{Fe}_{20}$ (approximately $140 \mathrm{~nm}$ thick) layer and a ferrimagnetic $\mathrm{Dy}_{20} \mathrm{Co}_{80}$ (approximately $70 \mathrm{~nm}$ thick) layer, denoted as $\mathrm{Dy}-\mathrm{Co} / \mathrm{Ni}-\mathrm{Fe}$, is prepared on a $\mathrm{Si}$ wafer [Fig. 2(a)]. In this system Dy-Co possesses a large perpendicular magnetic anisotropy, while the soft magnetic Ni-Fe layer shows an easy in-plane behavior. During the deposition of the individual layers, a small in-plane magnetic field of $\mu_{0} H=5 \mathrm{mT}$ is applied to establish a uniaxial magnetic anisotropy for the Ni-Fe layer in the film plane. The easy axis of Ni-Fe is denoted in the following as the $x$ axis. At room temperature the ferrimagnetic Dy-Co layer is in the Co-dominant phase $\left(M_{S}{ }^{\mathrm{Co}}>M_{S}{ }^{\mathrm{Dy}}\right)$ and the magnetic moments of $\mathrm{Co}$ and $\mathrm{Ni}-\mathrm{Fe}$ are ferromagnetically coupled at the interface.

The magnetization behavior of the Dy-Co/Ni-Fe bilayer is investigated at room temperature using the MOKE with the magnetic field applied perpendicular (PMOKE; H parallel to the $z$ axis) and parallel (LMOKE; H parallel to the $x$ and $y$ axes) to the sample plane. Figure 2(d) shows the out-of-plane behavior with a coercive field of $\mu_{0} H_{z} \approx \pm 120 \mathrm{mT}$, revealing the switching of the magnetization of the Dy-Co layer. After the sample is magnetized along the surface normal with $\mu_{0} H_{z}=-1 \mathrm{~T}$, the bilayer is studied by LMOKE. When the external magnetic field is applied along the $x$ axis, a positive shift of the hysteresis loop by $\mu_{0} H_{\mathrm{EB}}=39 \mathrm{mT}$ is observed, confirming the occurrence of an in-plane EB effect in the bilayer (blue curve). If the in-plane magnetic field is applied, however, perpendicular to the $x$ axis, no exchange-bias effect is found, and the hysteresis loop exhibits a magnetic-hardaxis behavior (black curve). When the sample is magnetized with an opposite out-of-plane field $\left(\mu_{0} H_{z}=1 \mathrm{~T}\right)$, the $M_{x}\left(H_{x}\right)$ hysteresis is shifted to negative field values (red curve), while the absolute value of the exchange-bias field $H_{\mathrm{EB}}$ remains the same. This demonstrates that the direction of the EB effect can be switched by solely reversing the direction of the out-of-plane magnetization of Dy-Co.
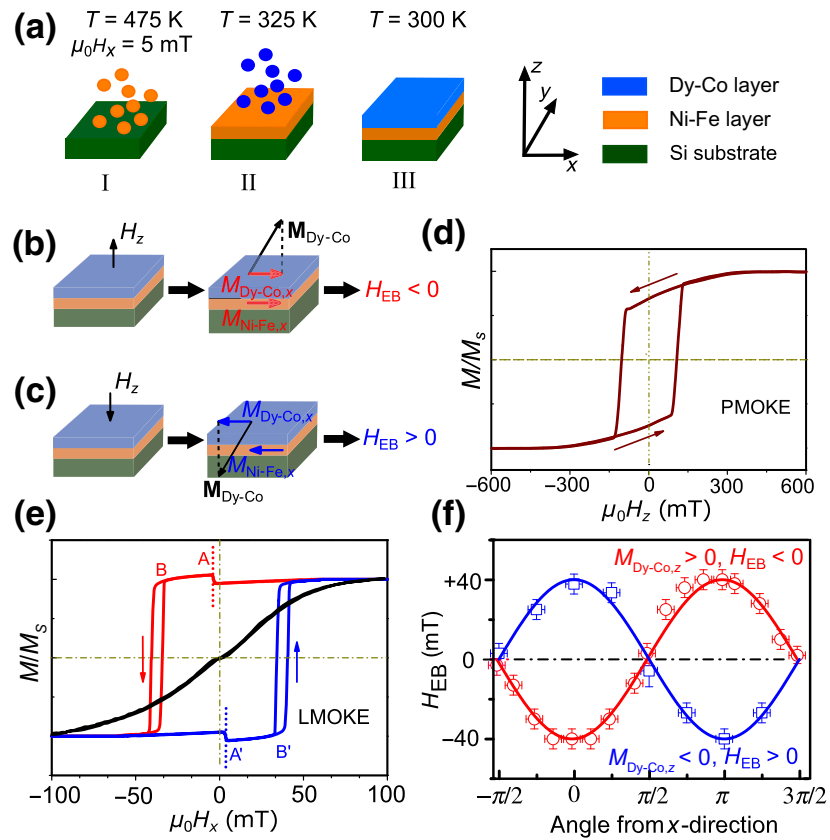

FIG. 2. Chirality-created tunable EB effect in Dy-Co/Ni-Fe. (a) Sample preparation: the Ni-Fe layer is deposited on a heated $\mathrm{Si}$ substrate $(T=475 \mathrm{~K})$ at a magnetic in-plane field of $5 \mathrm{mT}(\mathrm{I})$, followed by the deposition of Dy-Co at $T=325 \mathrm{~K}$ (II), and the magnetic characterization is performed at room temperature (III). (b),(c) The direction of the in-plane EB in Dy-Co/Ni-Fe depends on the perpendicular component of magnetization of Dy-Co [(b) $M_{\mathrm{Dy}-\mathrm{Co}, z}>0, H_{\mathrm{EB}}<0$; (c) $M_{\mathrm{Dy}-\mathrm{Co}, z}<0, H_{\mathrm{EB}}>0$ ]. (d) Out-ofplane magnetic hysteresis loop ( $\mathbf{H}$ parallel to the $z$ axis) and (e) in-plane hysteresis loops for $\mathbf{H}$ perpendicular to the $x$ axis (black) and parallel to the $x$ axis ( $\operatorname{red} M_{\mathrm{Dy}-\mathrm{Co}, z}>0$ and blue $\left.M_{\mathrm{Dy}-\mathrm{Co}, z}<0\right)$. The unidirectional shifts in the switching field for $\mathrm{Ni}-\mathrm{Fe}$ and Dy-Co are marked as $A$ and $B$, or $A^{\prime}$ and $B^{\prime}$. (f) Azimuthalangle dependence of the EB field after premagnetization with $\mu_{0} H_{z}=-1 \mathrm{~T}$ (blue curve) and $\mu_{0} H_{z}=1 \mathrm{~T}$ (red curve), respectively. The solid lines are $\pm \cos \phi$ curves fitted to the data.

For switching of the EB direction, a magnetic field has to be applied along the out-of-plane direction that is sufficient to completely reverse $M_{z}$ of the Dy-Co layer; that is, the field has to be slightly larger than the coercive field of $\left|\mu_{0} H_{z}\right|=120 \mathrm{mT}$. Once established, the EB persists unaltered even after application of magnetic fields of more than $\mu_{0} H= \pm 6 \mathrm{~T}$ in the film plane. Figure 2(f) shows the azimuthal-angle dependence of the EB field taken after the sample was magnetized with $\mu_{0} H_{z}= \pm 1 \mathrm{~T}$. Within the error bar, the azimuthal behavior of the EB can be well fitted with a $\cos \phi$ function, with $\phi$ denoting the angle between the external field and the $x$ axis of the sample, and thus following the behavior of classic EB systems.

Besides the prominent magnetization reversal at $\mu_{0} H_{\mathrm{EB}}= \pm 39 \mathrm{mT}$, marked as $B$ and $B^{\prime}$ in Fig. 2(e) for both premagnetized states, additional kinks are observed at low magnetic fields (approximately $\pm 3 \mathrm{mT}$ ), marked as $A$ and $A^{\prime}$. The kinks correspond to the switching of the Ni-Fe 
layer, and hence a dual exchange-bias effect exists for the switching of the bilayer. The signs of the magneto-optical constants of Ni-Fe and Dy-Co are opposite each other, so it wrongly appears that the Ni-Fe layer switches antiparallel to the field. This misleading feature does not occur for the SQUID technique. SQUID measurements are applied to gain complementary information on the magnetization behavior of the bilayer system, since the MOKE is limited in penetration depth and is therefore mainly sensitive to the Dy-Co layer.

Figure 3(a) shows the in-plane SQUID hysteresis curve for premagnetization of $\mu_{0} H_{z}=-1 \mathrm{~T}$ with its main signal slightly shifted with respect to zero magnetic field, in absolute accordance with the position of the small kink at $A^{\prime}$ present in the LMOKE measurement. The SQUID signal is dominated by the magnetization of the Ni-Fe layer, which is much larger than the magnetization of the ferrimagnetic Dy-Co layer, confirming that the kinks at $A^{\prime}$ originate from the magnetic switching of the Ni-Fe layer. However, a tiny kink reflecting the switching of the Dy-Co at $\mu_{0} H_{x}=39 \mathrm{mT}$ is also observed in the SQUID measurements, confirming the presence of the dual EB effect. From the results for the dual EB (Figs. 2 and 3(a)) and the comparison with Fig. 1, we can finally conclude that for Dy$\mathrm{Co} / \mathrm{Ni}-\mathrm{Fe}$ a clockwise chirality is favored for the interfacial domain wall.

\section{FIELD-DEPENDENT MAGNETIC STRUCTURES}

To understand the underlying spin structure providing the EB effect in more detail, we use PNR measurements, which enable us to probe the nuclear and magnetization depth profiles for the different magnetic states [44-46]. According to the results of the LMOKE and SQUID measurements, the Dy-Co layer has a slightly tilted magnetic configuration in the $x-z$ plane, while the magnetic moments of the Ni-Fe layer are aligned along the $x$ axis. Considering the different in-plane switching fields for Dy-Co and $\mathrm{Ni}-\mathrm{Fe}$, there are three principal field-dependent magnetic states in the bilayer, marked as I, II, and III in Fig. 3(a). For these states, the details of the nuclear profile and the magnetization-vector configuration were revealed from the analysis of the non-spin-flip reflectivites $R^{++}$(spin-up neutrons) and $R^{--}$(spin-down neutrons) and the corresponding spin-asymmetry ratios $\left(R^{++}-R^{--}\right) /\left(R^{++}+\right.$ $R^{--}$) shown in Figs. 3(b) and 3(c), respectively. The corresponding profiles of the scattering-length density (both nuclear and magnetic) are shown in Fig. 3(d). The nonsharp transitions of the nuclear scattering-length density at the surface, Dy-Co/Ni-Fe interface, and Ni-Fe/Si interface are due to the roughnesses, which are determined to be 2.0, 2.2 , and $1.6 \mathrm{~nm}$, respectively, from the fittings. (a)

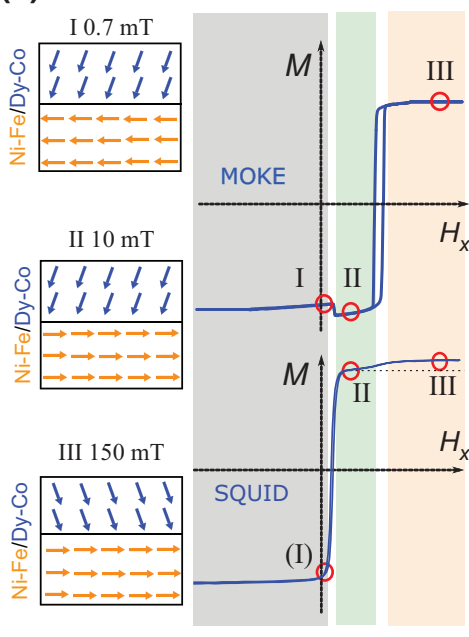

(b)

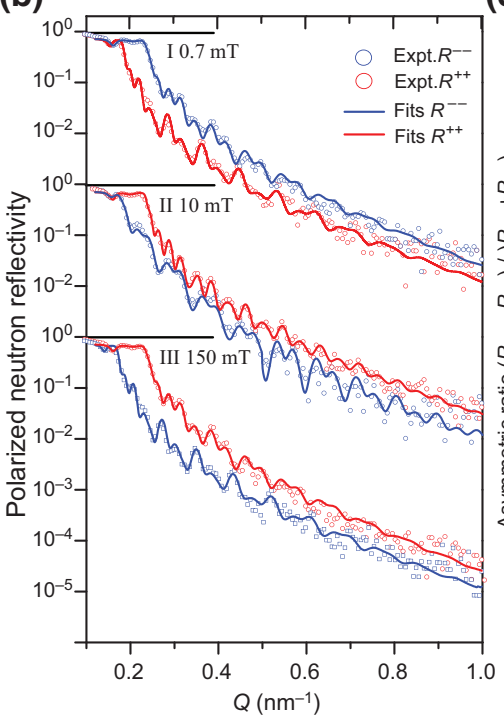

(c)

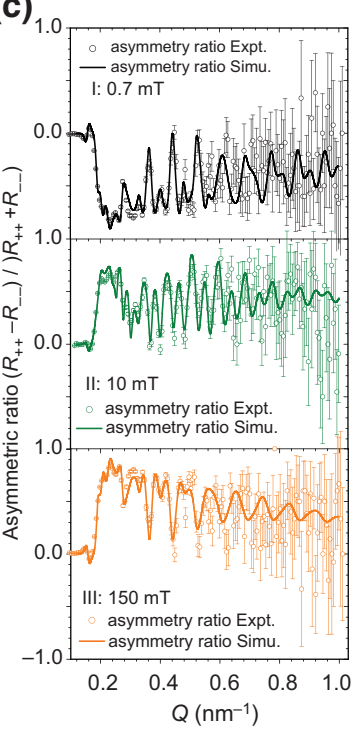

(d)

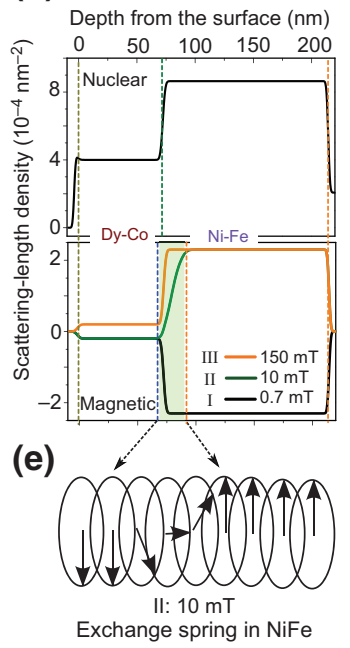

FIG. 3. Chiral interfacial domain wall. (a) Three different magnetic states marked as I, II, and III are obtained from the MOKE and SQUID hysteresis loops. (b) PNR data (expt.), and the results of the simulation (fits) taken at in-plane fields of $\mu_{0} H_{x}=0.7,10$, and 150 $\mathrm{mT}$ for spin-up neutrons (red) and spin-down neutrons (blue), representing the three states given in (a) obtained after the sample was premagnetized at a field of $\mu_{0} H_{z}=-1 \mathrm{~T}$. (c) Spin-asymmetry ratio $\left(R^{++}-R^{--}\right) /\left(R^{++}+R^{--}\right)$for the three spin states. (d) Nuclear and magnetic scattering-length density profiles of Dy-Co/Ni-Fe layers obtained from the simulation. Magnetic states I, II, and III are clearly observed, and are well separated by the two reversible kinks, at $\mu_{0} H_{\mathrm{EB}}=3$ and $39 \mathrm{mT}$. For state II, the transition regime in the magnetic-scattering-length density data (green area) indicates an exchange springlike spin texture (only the in-plane component) at the interface as depicted in (e) for $3<\mu_{0} H_{x}<39 \mathrm{mT}$. 
First, a small in-plane magnetic field of $+0.7 \mathrm{mT}$ is applied along the $x$ axis after the initial out-of-plane magnetic field of $-1 \mathrm{~T}$ was switched off (state I). A simultaneous fit to the experimental reflectivity with opposite polarization states $\left(R^{++}\right.$and $\left.R^{--}\right)$shows that the in-plane component of magnetization of the Dy-Co layer and that of the Ni-Fe layer are magnetized along the same inplane direction, antiparallel to the direction of the external field, in agreement with SQUID and MOKE measurements [Fig. 3(a)]. The assumption of a constant, uniform in-plane magnetization inside both layers leads to a very good agreement between the experimental and theoretical curves. The result at quasizero magnetic field demonstrates that the system has a preference for a certain in-plane orientation of magnetization after it has been premagnetized along one of the out-of-plane directions.

At $\mu_{0} H_{x}=10 \mathrm{mT}$ (state II), clear changes in the polarized-reflectivity curves are observed. The fit to the experimental data shows that the in-plane magnetization direction of the Ni-Fe has switched along the direction of the field, while the in-plane component of Dy-Co magnetization remains orientated in the previous, opposite direction. For this oppositely aligned magnetic layer configuration, PNR measurements reveal the existence of an approximately-20-nm-thick interfacial region (much larger than the interface roughness of $2.2 \mathrm{~nm}$ ) where the in-plane magnetization gradually changes from opposite to field-magnetized Dy-Co to field-aligned magnetization of Ni-Fe. This indicates the formation of an interfacial layer where the magnetic moments are perpendicularly oriented to the applied magnetic field as is the case for noncollinear spin structures such as exchange spring structures [Fig. 3(e)], similarly to what was observed in a Co-Pt/permalloy/Ta/permalloy heterostructure [46]. After increase of the external magnetic field above $H_{\mathrm{EB}}$ of the Dy-Co layer $\left(\mu_{0} H_{x}=150 \mathrm{mT}\right.$, state III), the PNR results reveal that the in-plane components of the magnetization of both layers are field aligned. The magnetization profile around the interface of both layers again follows the chemical profile.

From the complementary results from the MOKE, SQUID, and PNR techniques, the scenario for the presence of a chiral interfacial domain wall with clockwise chirality as depicted in Fig. 1(c) is confirmed. During the initial application of an external magnetic field along the out-of-plane direction $\left(\left|\mu_{0} H_{z}\right|>120 \mathrm{mT}\right)$, the magnetization of the Dy-Co and Ni-Fe layers follows the applied field and the $z$ component of Dy-Co switches along the field direction. After the magnetic field is switched off, the Ni-Fe moments follow their in-plane uniaxial magnetic anisotropy, turning their moments along the negative or positive $x$ axis depending on the orientation of $M_{\mathrm{Dy}-\mathrm{Co}, z}$. The PNR data, with the absence of the off-specular scattering, clearly demonstrate that such a single-domain state is created. The resulting Dy-Co moments, which are exchange coupled at the interface to the $\mathrm{Ni}-\mathrm{Fe}$ layer, partly follow the Ni-Fe moments into the in-plane direction, leading to a tilted configuration. By application of an in-plane magnetic field along the opposite direction of the orientation of the Ni-Fe and Dy-Co moments, first the Ni-Fe moments start to reverse, slightly exchange biased, until the in-plane orientations of $\mathrm{Ni}-\mathrm{Fe}$ and Dy-Co are antiparallel aligned [state I to state II in Fig. 3(a)]. At this stage the magnetic configuration at the interface leads to an interfacial regime that can be pictured as an exchange spring structure. When the magnetic field exceeds the larger EB field of $39 \mathrm{mT}$, the in-plane magnetization of the Dy-Co layer finally also reverses and aligns along the field direction as depicted in state III in Fig. 3(a), resolving the spring structure at the interface.

\section{ORIGIN OF THE CHIRAL DOMAIN WALL}

During the growth of the Dy-Co alloy onto the NiFe layer, the latter is in a single-domain state with the moments pointing along the $x$ axis due to the applied field of $\mu_{0} H_{x}=5 \mathrm{mT}$. Because of the ferromagetic coupling between $\mathrm{Co}$ and $\mathrm{Ni}-\mathrm{Fe}$ moments, the Co moments are slightly tilted away from the perpendicular easy axis along the $x$ direction with the option to point upward (state A) or downward (state B), respectively (upper panel in Fig. 4). Since the presence of the EB effect revealed that a clockwise chirality of the interfacial domain wall is favored, the Co moments tilt upward. Because of the cone spin structure of Dy [47] and its weak coupling to the Ni-Fe layer, the averaged in-plane magnetic component of Dy is relatively small and, for clarity, is ignored in the following discussion as depicted in Fig. 4. Hence, the magnetic moments of Co and Dy atoms at neighboring atomic sites $i$ and $j$ can be written as $\mathbf{m}_{i}=a_{1} \vec{x}+c_{1} \vec{z}$ and $\mathbf{m}_{j}=c_{2} \vec{z}$, respectively, with $a_{1}, c_{1}$, and $c_{2}$ representing the Co and Dy magnetic components along the $+x$ and $+z$ axes.

To understand the origin of the EB effect, a possible DMI effect between Co and Dy moments is considered, whose contribution to the free energy is generally written as $E_{\mathrm{DM}} \propto \mathbf{D}_{i j} \cdot\left(\mathbf{S}_{i} \times \mathbf{S}_{j}\right)$ where $\mathbf{D}_{i j}$ is the DM vector and $\mathbf{S}_{i}$ and $\mathbf{S}_{j}$ are the respective spins [20,21]. In consideration of a homogeneous, nonzero DM vector component along the $+y$ axis $\left(D_{i j, y}>0\right)$ or the $-y$ axis $\left(D_{i j, y}<0\right)$ axis, the contribution of the DMI effect to the free energy is $a_{1} c_{2} D_{i j, y}$, lifting the degeneracy between state A and state B shown in Fig. 4. Therefore, a preferred tilting of the magnetization with an angle of $\theta$ or $-\theta$ with respect to the film normal exists, and the sign of the angle depends on whether $D_{i j, y}$ points along the $-y$ axis or the $+y$ axis. All other projections of the DM vector cannot give any contribution to the preferred chirality.

Because of the ferromagnetic exchange coupling between the $\mathrm{Co}$ and Ni-Fe moments, the chiral effect in 


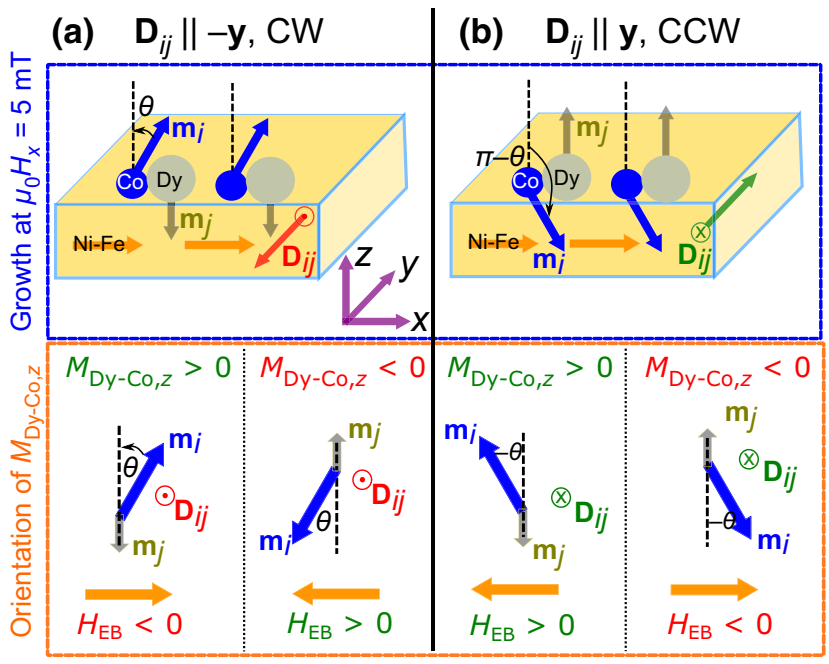

FIG. 4. Microscopic origin of the chirality-created tunable EB effect. During the growth process, the application of an inplane field $\mu_{0} H_{x}=5 \mathrm{mT}$ provides a uniaxial anisotropy and a field-aligned single-domain state in Ni-Fe (top panel). With the ferromagnetic coupling between the $\mathrm{Co}$ and the $\mathrm{Ni}-\mathrm{Fe}$ atoms at the interface, two possible magnetization configurations may form when the Dy-Co layer is deposited on Ni-Fe; these are energetically degenerated in the case of vanishing DMI. The state depicted in (a) [or (b)] is favored in the case of a negative [positive] DMI constant $D_{i j, y}$ in Dy-Co along the $y$ direction ( $i$ is $\mathrm{Co}, j$ is Dy), correponding to a clockwise (CW) [counterclockwise $(\mathrm{CCW})$ ] chirality of the interfacial domain wall between $\mathrm{Co}$ and Ni-Fe. The sign of the perpendicular component of magnetization of the Dy-Co layer $M_{\mathrm{Dy}-\mathrm{Co}, z}\left(\mathrm{Co}\right.$ moments $\left.m_{i}\right)$ dictates the sign of the field $H_{\mathrm{EB}}$ (bottom panel). For $D_{i j, y}<0\left(D_{i j, y}>0\right)$ with CW $(\mathrm{CCW})$ chirality of interfacial DW, $M_{\mathrm{Dy}-\mathrm{Co}, z}$ and $H_{\mathrm{EB}}$ have opposite (same) signs.

Dy-Co is transferred to the moments ( $m_{\mathrm{Ni-Fe}}$ and $\left.m_{\mathrm{Co}}\right)$ at the interface and therefore a preferred chirality for the interfacial domain wall exists as depicted in the lower panel in Fig. 4. Without the presence of a macroscopic DMI during the growth proceess, the energies of clockwise and counterclockwise chiral spin structures at the interface are equal and no EB effect would be observed. For a clockwise chirality as favored for $\mathrm{Dy}-\mathrm{Co} / \mathrm{Ni}-\mathrm{Fe}$ the $\mathrm{DM}$ vector points along $-y$ direction.

To estimate the strength of the DMI, we take into account that a reverse field of $\mu_{0} H_{x}=39 \mathrm{mT}$ is required to generate the DMI-unfavored orientation between the Co and Dy spins [state III in Fig. 3(a) and state B in Fig. 4]. Hence, at this magnetic field the Zeeman energy overcompensates the DMI energy in the Dy-Co layer: $E=\mu_{0} H_{x} \Delta M_{S}^{\text {Dy-Co }}$. According to the SQUID data shown in Fig. 3(a) the in-plane component in Dy-Co changes by $\Delta M_{S}^{\text {Dy-Co }} \approx 65 \mathrm{kA} \mathrm{m}^{-1}$. Consequently, $E \approx 2.5 \mathrm{~kJ} \mathrm{~m}^{-3}$ results for the bulk DMI energy density between Dy and Co moments. The remaining question is whether the DMI is inherently present in the whole Dy-Co layer [48] or exists only in the Dy-Co atomic layers next to the interface [19] triggered by the adjacent Ni-Fe layer. Very recently, a bulk DMI in $(\mathrm{Gd}, \mathrm{Fe})$, Co amorphous ferrimagnets has been reported, and was attributed to an asymmetric distribution of the elemental content [48]. Even though no evidence can be provided here for such an asymmetric distribution of Dy and Co, the finding of an asymmetric magnetization from the surface to the bulk in a thin film of $\mathrm{DyCo}_{4}$ [18] suggests the existence of a bulklike DMI in the DyCo system. The effective DMI constant can be estimated to be $D_{\text {eff }}=E t=0.18 \mathrm{~mJ} \mathrm{~m}^{-2}$, with $t$ the thickness of the Dy-Co layer of $70 \mathrm{~nm}$. The value of $0.18 \mathrm{~mJ} \mathrm{~m}^{-2}$ follows the value for $(\mathrm{Gd}, \mathrm{Fe})$,Co when extrapolated to $70 \mathrm{~nm}$ [48]. This value is one order of magnitude smaller than for the prototypical $\mathrm{Pt} / \mathrm{Co}$ interface (on the order of millijoules per square meter) [49], in which Néel domain walls or skyrmions are stabilized [50,51]. Even though $D_{\text {eff }}$ is much smaller here, the value is significant since the whole interfacial area contributes to the DMI energy and thus to the observed chirality effect. To distinguish between bulk and interface DMI effects, one has to determine the DMI strength as a function of Dy-Co thickness in future studies.

The DMI-induced chiral interfacial domain wall is not the only mandatory requirement for generating the EB effect in Dy-Co/Ni-Fe. As illustrated in Fig. 5, the stability of the out-of-plane magnetic component is also required; in particular, it may not reverse under the application of an in-plane magnetic field. As shown in Fig. 5(a), a dual in-plane exchange-bias effect exists when a single-domain state is formed in the Dy-Co layer. In contrast, in the case of a multidomain state with up and down domains in the Dy-Co layer, which, for example, can be generated by the application of a reversed out-of-plane magnetic field equal to the coercive field, a double shifted in-plane hysteresis loop with two steps is observed [Fig. 5(b)]. The relative amount between both out-of-plane domains remains unchanged even after the application of a high magnetic in-plane field. Without this domain stability in the ferrimagnetic layer, the chirality-created EB effect described cannot be established on a laterally macroscopic scale. Such a case without domain stability is shown in Fig. 5(c), which depicts the in-plane hysteresis of a $\mathrm{Dy}_{26} \mathrm{Co}_{74} / \mathrm{Ni}-\mathrm{Fe}$ bilayer system. Here the out-of-plane magnetization of the $\mathrm{Dy}_{26} \mathrm{Co}_{74}$ layer, with compensation temperature and magnetic anisotropy different from those of $\mathrm{Dy}_{20} \mathrm{Co}_{80}[18,47$, 52-54], completely switches during the hysteresis, resulting in an axial symmetric loop without the presence of the EB effect. However, the fingerprint of the chiral DW at the Dy-Co/Ni-Fe interface (i.e., the occurrence of two steps in the hysteresis) still persists even for this alloy composition. The reason for the domain instability is the higher Dy concentration in the Dy-Co layer, which leads to a reduced perpendicular magnetic anisotropy at room temperature [52], so the Dy-Co magnetization is susceptible and can be 


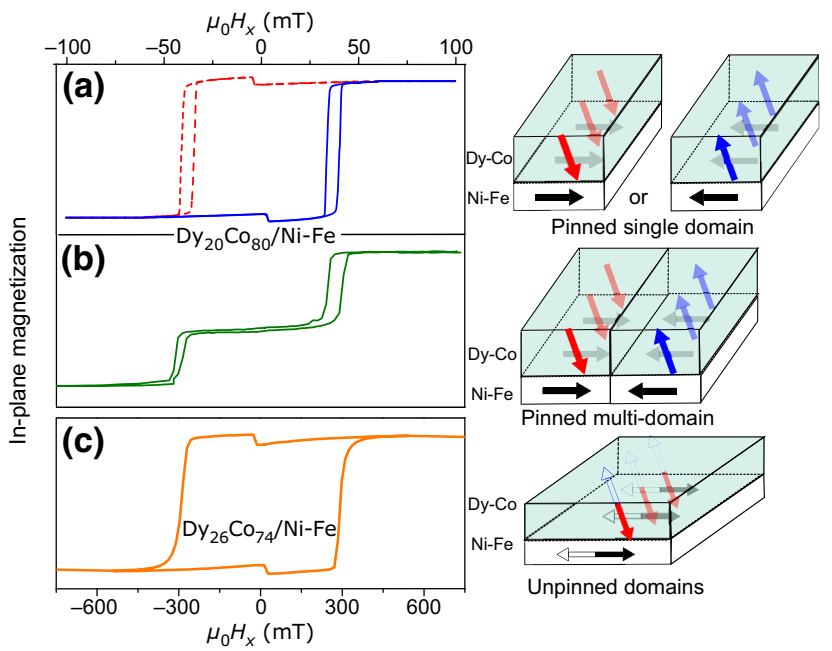

FIG. 5. Connection between the EB effect and domain stability. Three scenarios are depicted with the experimentally measured hysteresis loops (left) and the corresponding domain-state configurations (right). (a),(b) The results for the $\mathrm{Dy}_{20} \mathrm{Co}_{80} / \mathrm{Ni}-\mathrm{Fe}$ bilayer, (a) for the single-domain state with $M_{z \text {,Dy-Co }}<0$ (red) and $M_{z \text {,Dy-Co }}>0$ (blue), respectively, and (b) for the multidomain state where up and down domains exist simultaneously. (c) The scenario for a switching of $M_{\mathrm{Dy}-\mathrm{Co}, z}$ during the $H_{x}$-field application measured for a $\mathrm{Dy}_{26} \mathrm{Co}_{74} / \mathrm{Ni}-\mathrm{Fe}$ bilayer.

switched by the application of even low in-plane magnetic fields.

\section{CONCLUSION}

In conclusion, we report a tunable dual in-plane EB effect we observe at room temperature in the Dy-Co/NiFe bilayer system that is stable on a macroscopic scale up to high in-plane magnetic fields $\left(\mu_{0} H \leq 6 \mathrm{~T}\right)$. The direction of the EB effects can be switched and controlled by the direction of a moderate magnetic field applied along the out-of-plane direction beforehand. The results from complementary MOKE, SQUID, and PNR measurements lead to the conclusion that the EB effect originates from the chiral spin texture of the interfacial domain wall. The creation of the chiral interfacial domain wall is a consequence of the interplay between DMI in Dy-Co and ferromagnetic exchange coupling between the $\mathrm{Ni}-\mathrm{Fe}$ and Dy-Co layers, whose uniaxial anisotropies are oriented orthogonally to each other. Realizations of isothermal EB switching were reported earlier by means of local electric fields [55-62] and magnetic fields [63,64]. Here the chirality-created tunable dual EB effect may provide an approach to overcome the limitations of conventional EB systems. The use of an easy and fast production path via standard sputtering techniques to create a chiral domain wall for the establishment of a sizable exchange-bias effect makes this film system very attractive for application in future spintronic devices. Particularly, the flexibility in controlling exchange bias at room temperature could be exploited in future spintronic devices and new concepts where, for example, the storage density can be increased, allowing more than the standard dual logical states, with thermomagnetic data erasing and writing $[65,66]$. Besides, the extreme stability of the exchange-bias effect even at very high magnetic fields without any observable training effect is a very important feature for the fabrication of long-lived spintronic devices. Finally, the creation of chiral interfacial domain walls in thin-film systems provides a possible way to control the out-of-plane magnetization by the application of an in-plane magnetic field. However, compared with the frequently discussed chiral spin textures of skyrmions, further investigations on the dynamics and transport properties of such chiral interfacial domain walls are required to determine their full potential for technological applications.

\section{APPENDIX: SAMPLE PREPARATION AND CHARACTERIZATION}

The $\mathrm{Dy}_{20} \mathrm{Co}_{80} / \mathrm{Ni}_{80} \mathrm{Fe}_{20}$ film is fabricated by thermal evaporation in a vacuum of $3 \times 10^{-10}$ Torr by successive sputtering of the Ni-Fe and Dy-Co layers from independent evaporators (Ni-Fe, Dy, Co) with a ring cathode onto $\mathrm{Si}(110)$ substrates. Ni-Fe is deposited on $\mathrm{Si}(110)$ at $T=475 \mathrm{~K}$ at $\mu_{0} H=5 \mathrm{mT}$ applied along the $x$ axis, DyCo is co-deposited at $T=325 \mathrm{~K}$, and the Dy-Co/Ni-Fe bilayer is finally cooled to room temperature. The deposition rates are identical at $0.5 \mathrm{~nm} \mathrm{~s}^{-1}$. In good agreement with the PNR fittings, the thicknesses of the Ni-Fe and Dy-Co determined by x-ray-reflectivity measurements are $139 \pm 5 \mathrm{~nm}$ and $70 \pm 2 \mathrm{~nm}$, respectively. Since for DyCo the room-temperature-compensation composition is between $\mathrm{Dy}_{21} \mathrm{Co}_{79}$ and $\mathrm{Dy}_{24} \mathrm{Co}_{76}[18,47,53,54]$, the net magnetization in the ferrimagnetic $\mathrm{Dy}_{20} \mathrm{Co}_{80}$ is $M^{\mathrm{Co}}-$ $M^{\text {Dy }}$. The in-plane magnetic field applied during the sample preparation that imprints the uniaxial anisotropy is required for the EB effect. In similar samples prepared without a field during the growth of the films, no preferred in-plane tilting direction is established, resulting in the absence of a macroscopically observable EB effect.

PNR measurements were applied to investigate the magnetic depth profile of the sample and, in particular, to obtain depth-dependent information about the changes in the magnetic structure for the different magnetic states. The PNR measurements are performed at the Super-ADAM reflectometer at the Institut Laue-Langevin in collaboration with the University of Uppsala and on the Magnetism Reflectometer (beamline BL-4A) [67] at the Spallation Neutron Source of Oak Ridge National Laboratory. The PNR spectra are taken for several external magnetic fields applied along the $x$ axis of the sample using polarization analysis, in particular to extract non-spin-flip intensities that are sensitive to the magnetic moments along 
the applied magnetic guide field; that is, $R^{++}$and $R^{--}$ for the magnetic moments aligned parallel and antiparallel to the applied in-plane field, respectively. PNR is sensitive only to magnetic moments perpendicular to the momentum transfer vector $\mathbf{Q}$ of the measurement (i.e., only the in-plane magnetization is detected). The measured reflectivity curves are analyzed using the method of Parratt [68]. Before the PNR measurements, the sample is premagnetized in a magnetic field of $\mu_{0} H=-1 \mathrm{~T}$ in the out-of-plane direction to establish the EB effect as observed in the MOKE measurements.

The nuclear scattering-length densities are $3.95 \times$ $10^{-4} \mathrm{~nm}^{-2}$ for the Dy-Co layer and $8.6 \times 10^{-4} \mathrm{~nm}^{-2}$ for the Ni-Fe layer. The magnetic moments can be calculated from the magnetic scattering-length densities of the fits. A magnetic scattering-length density of $(2.05 \pm 0.05) \times$ $10^{-4} \mathrm{~nm}^{-2}$ for the Ni-Fe layer corresponds to a magnetic moment of $0.96 \mu_{B}$ per Ni-Fe formula unit, while Dy-Co, due to its ferrimagnetic coupling between both sites, shows with a magnetic scattering-length density of $(0.45 \pm 0.05) \times 10^{-4} \mathrm{~nm}^{-2}$ a much lower magnetization of $0.2 \mu_{B}$ per Dy-Co formula unit.

\section{ACKNOWLEDGMENTS}

K.C. benefited from the support of the Deusche Forschungsgemeinschaft (DFG) via Project No. 615811. A.P.-K. gratefully acknowledges support from the DFG via Sonderforschungsbereich (collaborative research center) SFB925 (subproject B3).

[1] W. H. Meiklejohn and C. P. Bean, New magnetic anisotropy, Phys. Rev. 102, 1413 (1956).

[2] S. Parkin, J. Xin, C. Kaiser, A. Panchula, K. Roche, and M. Samant, Magnetically engineered spintronic sensors and memory, Proc. IEEE 91, 661 (2003).

[3] C. Chappert, A. Fert, and F. N. V. Dau, The emergence of spin electronics in data storage, Nat. Mater. 6, 813 (2007).

[4] J. Nogues and I. K. Schuller, Exchange bias, J. Magn. Magn. Mater. 192, 203 (1999).

[5] M. Kiwi, Exchange bias theory, J. Magn. Magn. Mater. 234, 584 (2001).

[6] H. Ohldag, F. Scholl, A. Nolting, E. Arenholz, S. Maat, A. T. Young, M. Carey, and J. Stohr, Correlation between Exchange Bias and Pinned Interfacial Spins, Phys. Rev. Lett. 91, 017203 (2003).

[7] F. Radu, S. K. Mishra, I. Zizak, A. I. Erko, H. A. Dürr, W. Eberhardt, G. Nowak, S. Buschhorn, H. Zabel, K. Zhernenkov, M. Wolff, D. Schmitz, E. Schierle, E. Dudzik, and R. Feyerherm, Origin of the reduced exchange bias in an epitaxial FeNi(111)/CoO(111) bilayer, Phys. Rev. B 79, 184425 (2009).

[8] S. K. Mishra, F. Radu, S. Valencia, D. Schmitz, E. Schierle, H. A. Dürr, and W. Eberhardt, Dual behavior of antiferromagnetic uncompensated spins in $\mathrm{NiFe} / \mathrm{IrMn}$ exchange biased bilayers, Phys. Rev. B 81, 212404 (2010).
[9] J. Wu, J. S. Park, W. Kim, E. Arenholz, M. Liberati, A. Scholl, Y. Z. Wu, C. Hwang, and Z. Q. Qiu, Direct Measurement of Rotatable and Frozen $\mathrm{CoO}$ Spins in Exchange Bias System of $\mathrm{CoO} / \mathrm{Fe} / \mathrm{Ag}(001)$, Phys. Rev. Lett. 104, 217204 (2010).

[10] R. Morales, A. C. Basaran, J. E. Villegas, D. Navas, N. Soriano, B. Mora, C. Redondo, X. Batlle, and I. K. Schuller, Exchange-Bias Phenomenon: The Role of the Ferromagnetic Spin Structure, Phys. Rev. Lett. 114, 097202 (2015).

[11] F. Hellman, R. B. van Dover, and E. M. Gyorgy, Unexpected unidirectional anisotropy in amorphous $\mathrm{Tb}-$ Fe/Ni-Fe-Mo bilayer films, Appl. Phys. Lett. 50, 296 (1987).

[12] W. C. Cain and M. H. Kryder, Investigation of the exchange mechanism in NiFe-TbCo bilayers, J. Appl. Phys. 67, 5722 (1990).

[13] F. Radu, R. Abrudan, I. Radu, D. Schmitz, and H. Zabel, Perpendicular exchange bias in ferrimagnetic spin valves, Nat. Comm. 3, 715 (2012).

[14] A. K. Nayak, M. Nicklas, S. Chadov, P. Khuntia, C. Shekhar, A. Kalache, M. Baenitz, Y. Skourski, V. K. Guduru, A. Puri, U. Zeitler, J. M. D. Coey, and C. Felser, Design of compensated ferrimagnetic Heusler alloys for giant tunable exchange bias, Nat. Mater. 14, 679 (2015).

[15] S. M. Watson, T. Hauet, J. A. Borchers, S. Mangin, and E. E. Fullerton, Interfacial magnetic domain wall formation in perpendicular-anisotropy, exchange-spring films, Appl. Phys. Lett. 92, 202507 (2008).

[16] C. Schubert, B. Hebler, H. Schletter, A. Liebig, M. Daniel, R. Abrudan, F. Radu, and M. Albrecht, Interfacial exchange coupling in $\mathrm{Fe}-\mathrm{Tb} /[\mathrm{Co} / \mathrm{Pt}]$ heterostructures, Phys. Rev. B 87, 054415 (2013).

[17] B. Hebler, P. Reinhardt, G. L. Katona, O. Hellwig, and M. Albrecht, Double exchange bias in ferrimagnetic heterostructures, Phys. Rev. B 95, 104410 (2017).

[18] K. Chen, D. Lott, F. Radu, F. Choueikani, E. Otero, and P. Ohresser, Observation of an atomic exchange bias effect in DyCo4 film, Sci. Rep. 5, 18377 (2015).

[19] R. Streubel, C.-H. Lambert, N. Kent, P. Ercius, A. T. N'Diaye, C. Ophus, S. Salahuddin, and P. Fischer, Experimental evidence of chiral ferrimagnetism in amorphous GdCo films, Adv. Mat. 30, 1800199 (2018).

[20] I. E. Dzyaloshinskii, Thermodynamical theory of 'weak' ferromagnetism in antiferromagnetic substances, Sov. Phys. JETP 5, 1259 (1957).

[21] T. Moriya, Anisotropic superexchange interaction and weak ferromagnetism, Phys. Rev. 120, 91 (1960).

[22] S. Dong, K. Yamauchi, S. Yunoki, R. Yu, S. Liang, A. Moreo, J.-M. Liu, S. Picozzi, and E. Dagotto, Exchange Bias Driven by the Dzyaloshinskii-Moriya Interaction and Ferroelectric Polarization at G-type Antiferromagnetic Perovskite Interfaces, Phys. Rev. Lett. 103, 127201 (2009).

[23] R. Yanes, J. Jackson, L. Udvardi, L. Szunyogh, and U. Nowak, Exchange Bias Driven by Dzyaloshinskii-Moriya Interactions, Phys. Rev. Lett. 111, 217202 (2013).

[24] R. M. Matthias Hudl and P. Nordblad, Tunable exchange bias in dilute magnetic alloys-chiral spin glasses, Sci. Rep. 6, 19964 (2016). 
[25] M. Cubukcu, J. Sampaio, K. Bouzehouane, D. Apalkov, A. V. Khvalkovskiy, V. Cros, and N. Reyren, DzyaloshinskiiMoriya anisotropy in nanomagnets with in-plane magnetization, Phys. Rev. B 93, 020401 (2016).

[26] D.-S. Han, N.-H. Kim, J.-S. Kim, Y. Yin, J.-W. Koo, J. Cho, S. Lee, M. Klui, H. J. M. Swagten, B. Koopmans, and C.Y. You, Asymmetric hysteresis for probing DzyaloshinskiiMoriya interaction, Nano Lett. 16, 4438 (2016).

[27] S. Pizzini, J. Vogel, S. Rohart, L. D. Buda-Prejbeanu, E. Jue, O. Boulle, I. M. Miron, C. K. Safeer, S. Auffret, G. Gaudin, and A. Thiaville, Chirality-induced Asymmetric Magnetic Nucleation in $\mathrm{Pt} / \mathrm{Co} / \mathrm{AlO}_{x}$ Ultrathin Microstructures, Phys. Rev. Lett. 113, 047203 (2014).

[28] M. Heide, G. Bihlmayer, and S. Blügel, DzyaloshinskiiMoriya interaction accounting for the orientation of magnetic domains in ultrathin films: Fe/W(110), Phys. Rev. B 78, 140403 (2008).

[29] T. A. Moore, I. M. Miron, G. Gaudin, G. Serret, S. Auffret, B. Rodmacq, A. Schuhl, S. Pizzini, J. Vogel, and M. Bonfim, High domain wall velocities induced by current in ultrathin $\mathrm{Pt} / \mathrm{Co} / \mathrm{AlO}_{x}$ wires with perpendicular magnetic anisotropy, Appl. Phys. Lett. 93, 262504 (2008).

[30] A. Thiaville, S. Rohart, E. Jue, V. Cros, and A. Fert, Dynamics of Dzyaloshinskii domain walls in ultrathin magnetic films, Europhys. Lett. 100, 57002 (2012).

[31] G. Chen, J. Zhu, A. Quesada, J. Li, A. T. N'Diaye, Y. Huo, T. P. Ma, Y. Chen, H. Y. Kwon, C. Won, Z. Q. Qiu, A. K. Schmid, and Y. Z. Wu, Novel Chiral Magnetic Domain Wall Structure in $\mathrm{Fe} / \mathrm{Ni} / \mathrm{Cu}(001)$ Films, Phys. Rev. Lett. 110, 177204 (2013).

[32] S. Emori, U. Bauer, S. Ahn, E. Martinez, and G. S. D. Beach, Current-driven dynamics of chiral ferromagnetic domain walls, Nat. Mater. 12, 611 (2013).

[33] O. Boulle, S. Rohart, L. D. Buda-Prejbeanu, E. Jue, I. M. Miron, S. Pizzini, J. Vogel, G. Gaudin, and A. Thiaville, Domain Wall Tilting in the Presence of the DzyaloshinskiiMoriya Interaction in Out-of-Plane Magnetized Magnetic Nanotracks, Phys. Rev. Lett. 111, 217203 (2013).

[34] A. Brataas, Spintronics: Chiral domain walls move faster, Nat. Nanotechnol. 8, 485 (2013).

[35] J. Torrejon, J. Kim, J. Sinha, S. Mitani, M. Hayashi, M. Yamanouchi, and $\mathrm{H}$. Ohno, Interface control of the magnetic chirality in $\mathrm{CoFeB} / \mathrm{MgO}$ heterostructures with heavy-metal underlayers, Nat. Comm. 5, 4655 (2014).

[36] K. Ryu, L. Thomas, S. Yang, and S. Parkin, Chiral spin torque at magnetic domain walls, Nat. Nanotechnol. 8, 527 (2013).

[37] G. Chen, T. Ma, A. T. N’Diaye, H. Kwon, C. Won, Y. $\mathrm{Wu}$, and A. K. Schmid, Tailoring the chirality of magnetic domain walls by interface engineering, Nat. Comm. 4, 2671 (2013).

[38] T. N. A. Nguyen, Y. Fang, V. Fallahi, N. Benatmane, S. M. Mohseni, R. K. Dumas, and J. Akerman, [Co/Pd]-NiFe exchange springs with tunable magnetization tilt angle, Appl. Phys. Lett. 98, 172502 (2011).

[39] P. Saravanan, J.-H. Hsu, C. L. Tsai, C. Y. Tsai, Y. H. Lin, C. Y. Kuo, J.-C. Wu, and C.-M. Lee, Interplay between out-ofplane anisotropic L11-type CoPt and in-plane anisotropic $\mathrm{NiFe}$ layers in $\mathrm{CoPt} / \mathrm{NiFe}$ exchange springs, J. Appl. Phys. 115, 243905 (2014).
[40] A. Lisfi, J. C. Lodder, H. Wormeester, and B. Poelsema, Reorientation of magnetic anisotropy in obliquely sputtered metallic thin films, Phys. Rev. B. 66, 174420 (2002).

[41] M. Albrecht, G. Hu, I. L. Guhr, T. C. Ulbrich, J. Boneberg, P. Leiderer, and G. Schatz, Magnetic multilayers on nanospheres, Nat. Mater. 4, 203 (2005).

[42] L. You, O. Lee, D. Bhowmik, D. Labanowski, J. Hong, J. Bokor, and S. Salahuddin, Switching of perpendicularly polarized nanomagnets with spin orbit torque without an external magnetic field by engineering a tilted anisotropy, Proc. Natl. Acad. Sci 112, 10310 (2015).

[43] Ch. Binek, S. Polisetty, X. He, and A. Berger, Exchange Bias Training Effect in Coupled all Ferromagnetic Bilayer Structures, Phys. Rev. Lett. 96, 067201 (2006).

[44] C. Majkrzak, Polarized neutron reflectometry, Phys. B: Condens. Matt. 173, 75 (1991).

[45] S. J. Blundell, M. Gester, J. A. C. Bland, H. J. Lauter, V. V. Pasyuk, and A. V. Petrenko, Asymmetric Magnetization Reversal in Exchange-Biased Hysteresis Loops, Phys. Rev. Lett. 84, 3986 (2000).

[46] Y. Wang, X. He, T. Mukherjee, M. R. Fitzsimmons, S. Sahoo, and Ch. Binek, Magnetometry and transport data complement polarized neutron reflectometry in magnetic depth profiling, J. Appl. Phys., 110, 103914 (2011).

[47] K. Chen, D. Lott, F. Radu, F. Choueikani, E. Otero, and P. Ohresser, Temperature-dependent magnetic properties of ferrimagnetic $\mathrm{DyCo}_{3}$ alloy films, Phys. Rev. B 91, 024409 (2015).

[48] D. H. Kim, M. Haruta, H. W. Ko, G. Go, H. J. Park, T. Nishimura, D. Y. Kim, T. Okuno, Y. Hirata, Y. Futakawa, H. Yoshikawa, W. Ham, S. Kim, H. Kurata, A. Tsukamoto, Y. Shiota, T. Moriyama, S. B. Choe, K. J. Lee, and T. Ono, Bulk Dzyaloshinskii-Moriya interaction in amorphous ferrimagnetic alloys, Nat Mater. 18, 685 (2019).

[49] M. Belmeguenai, J. P. Adam, Y. Roussigne, S. Eimer, T. Devolder, J. V. Kim, S. M. Cherif, A. Stashkevich, and A. Thiaville, Interfacial Dzyaloshinskii-Moriya interaction in perpendicularly magnetized $\mathrm{Pt} / \mathrm{Co} / \mathrm{AlO}_{x}$ ultrathin films measured by Brillouin light spectroscopy, Phys. Rev. B 91, 180405 (2015).

[50] C. Moreau-Luchaire, C. Moutafis, N. Reyren, J. Sampaio, C. A. F. Vaz, N. Van Horne, K. Bouzehouane, K. Garcia, C. Deranlot, P. Warnicke, P. Wohlhüter, J.-M. George, M. Weigand, J. Raabe, V. Cros, and A. Fert, Additive interfacial chiral interaction in multilayers for stabilization of small individual skyrmions at room temperature, Nat Nanotechnol, 11, 444 (2016).

[51] F. Hellman et al., Interface-induced phenomena in magnetism, Rev. Mod. Phys. 89, 025006 (2017).

[52] D. Mergel, H. Heitmann, and P. Hansen, Pseudocrystalline model of the magnetic anisotropy in amorphous rare-earthtransition-metal thin films, Phys. Rev. B 47, 882 (1993).

[53] A. Agui, M. Mizumaki, T. Asahi, J. Sayama, K. Matsumoto, T. Morikawa, T. Matsushita, T. Osaka, and Y. Miura, Incident angle dependence of MCD at the Dy $\mathrm{M}_{5}$ edge of perpendicular magnetic $\mathrm{Dy}_{x} \mathrm{Co}_{100-x}$ films, J. Alloys Compd. 408-412, 741 (2006).

[54] A. Agui, M. Mizumaki, T. Asahi, K. Matsumoto, T. Morikawa, J. Sayama, and T. Osaka, Microscopic magnetic property of perpendicular magnetic films of $\mathrm{Dy}_{x} \mathrm{Co}_{100-x}$ 
measured using soft X-ray magnetic circular dichroism, J. Phys. Chem. Solids. 68, 2148 (2007).

[55] H. Ohno, D. Chiba, F. Matsukura, T. Omiya, E. Abe, T. Dietl, Y. Ohno, and K. Ohtani, Electric-field control of ferromagnetism, Nature 408, 944 (2000).

[56] P. Borisov, A. Hochstrat, X. Chen, W. Kleemann, and C. Binek, Magnetoelectric Switching of Exchange Bias, Phys. Rev. Lett. 94, 117203 (2005).

[57] X. He, Y. Wang, N. Wu, A. N. Caruso, E. Vescovo, K. D. Belashchenko, P. A. Dowben, and C. Binek, Robust isothermal electric control of exchange bias at room temperature, Nat. Mater. 9, 579 (2010).

[58] W. G. Wang, M. Li, S. Hageman, and C. L. Chien, Electricfield-assisted switching in magnetic tunnel junctions, Nat. Mater. 11, 64 (2012).

[59] S. M. Wu, S. A. Cybart, D. Yi, J. M. Parker, R. Ramesh, and R. C. Dynes, Full Electric Control of Exchange Bias, Phys. Rev. Lett. 110, 067202 (2013).

[60] F. A. Cuellar, Y. H. Liu, J. Salafranca, N. Nemes, E. Iborra, G. S. Santolino, M. Varela, M. G. Hernandez, J. W. Freeland, M. Zhernenkov, M. R. Fitzsimmons, S. Okamoto, S. J. Pennycook, M. Bibes, A. Barthelemy, S. G. E. te Velthuis, Z. Sefrioui, C. Leon, and J. Santa-Maria, Reversible electric-field control of magnetization at oxide interfaces, Nat. Comm. 5, 4215 (2014).

[61] F. Matsukura, Y. Tokura, and H. Ohno, Control of magnetism by electric fields, Nat. Nanotechnol. 10, 209 (2015).
[62] Y. W. Oh, S. C. Baek, Y. M. Kim, H. Y. Lee, K. D. Lee, C. G. Yang, E. S. Park, K. S. Lee, K. W. Kim, G. Go, J. R. Jeong, B. C. Min, H. W. Lee, K. J. Lee, and B. G. Park, Field-free switching of perpendicular magnetization through spin-orbit torque in antiferromagnet/ferromagnet/oxide structures, Nat. Nanotechnol 11, 878 (2016).

[63] S. Sahoo, T. Mukherjee, K. D. Belashchenko, and Ch. Binek, Isothermal low-field tuning of exchange bias in epitaxial $\mathrm{Fe} / \mathrm{Cr}_{2} \mathrm{O}_{3} / \mathrm{Fe}$, Appl. Phys. Lett. 91, 172506 (2007).

[64] Y. Shiratsuchi, K. Wakatsu, T. Nakamura, H. Oikawa, S. Maenou, Y. Narumi, K. Tazoe, C. Mitsumata, T. Kinoshita, H. Nojiri, and R. Nakatani, Isothermal switching of perpendicular exchange bias by pulsed high magnetic field, Appl. Phys. Lett. 100, 262413 (2012).

[65] V. A. Seredkin, G. I. Frolov, and V. Yu. Yakovchuk, Onedirectional magnetic anisotropy in $\mathrm{NiFe} / \mathrm{TbFe}$ layered film structure, Sov. Tech. Phys. Lett. 9, 621 (1983).

[66] V. A. Seredkin, S. V. Stolyar, G. I. Frolov, and V. Yu. Yakovchuk, Thermomagnetic data writing and erasing in DyCo/NiFe (TbFe/NiFe) film structures, Tech. Phys. Lett. 30, 820 (2004).

[67] V. Lauter, H. Ambaye, R. Goyette, W.-T. H. Lee, and A. Parizzi, Highlights from the magnetism reflectometer at the SNS, Phys. B: Condens. Matter. 404, 2543 (2009).

[68] L. G. Parratt, Surface studies of solids by total reflection of X-rays, Phys. Rev. B 95, 359 (1954). 